\title{
Alimentação de crianças durante a pandemia e as dificuldades dos responsáveis
}

\author{
Children's food during the pandemic and the difficulties of the responsible \\ Alimentación de niños durante la pandemia y las dificultades de los responsables
}

Recebido: 04/06/2021 | Revisado: 09/06/2021 | Aceito: 10/06/2021 | Publicado: 23/06/2021

Andressa De Jesus Peraira Faustino
ORCID: https://orcid.org/0000-0003-4942-5811
Enstituto Federal de Educação, Ciência e Tecnologia do Triângulo Mineiro, Brasil
E-mail: aj.pereirafaustino@ gmail.com
Letícia Vieira Castejon
ORCID: https://orcid.org/0000-0002-6615-0146
Instituto Federal de Educação, Ciência e Tecnologia do Triângulo Mineiro, Brasil
E-mail: leticiavieira@iftm.edu.br

\section{Resumo}

Neste trabalho abordaremos a dificuldade enfrentada pelas famílias atualmente diante o cenário da pandemia COVID19 para alimentação infantil de forma saudável e com variedades. Enfrentando as dificuldades financeiras e mediante o novo normal com trabalho HOME OFFICE e tendo as crianças em casa, de forma que além da demanda do trabalho, é necessário preparar a alimentação de suas crianças.

Palavra-chave: COVID-19; Alimentação saudável; Variedade na alimentação.

\begin{abstract}
In this paper, we will address the difficulty faced by families currently facing the COVID-19 scenario for healthy infant feeding with variety. Facing financial difficulties and through the new normal with HOME OFFICE work and having children at home, so that in addition to the demand for work, it is necessary to prepare the food for your children.

Keyword: COVID-19; Healthy food; Food variety.
\end{abstract}

\section{Resumen}

En este artículo, abordaremos la dificultad que enfrentan las familias que actualmente enfrentan el escenario pandémico COVID-19 para una alimentación infantil saludable con variedad. Enfrentando dificultades económicas y pasando por la nueva normalidad con el trabajo de DESPACHO A DOMICILIO y tener hijos en casa, por lo que además de la demanda de trabajo, es necesario preparar la comida para tus hijos.

Palabra clave: COVID-19; Dieta saludable; Variedad de alimentos.

\section{Introdução}

Em 2020, o Ministério da Saúde (MS) publicou um documento orientador e de instrução sobre a alimentação, ressaltando que novas publicações podem surgir, pois ainda pouco se sabe sobre a transmissão de Covid-19 por alimentos. Já se nota que as medidas adotada para a prevenção ao Covid-19 está alterando os hábitos alimentares, sociais e culturais das famílias.

Segundo Ribeiro-Silva e colaboradores (2020), o desafio atual é assegurar diferentes mecanismos que contribuam para garantir o direito humano à alimentação adequada e que fortaleçam as compras e as doações para a continuidade da operacionalização do PNAE (ajustado às demandas sanitárias da COVID-19) de forma mitigar a fome de diversos grupos vulneráveis no Brasil.

De acordo com o Fundo Nacional de Desenvolvimento da Educação - Ministério da Saúde (FNDE), o Programa Nacional de Alimentação Escolar (PNAE) oferece alimentação escolar e ações de educação alimentar e nutricional a estudantes de todas as etapas da educação básica pública. O governo federal repassa, a estados, municípios e escolas federais, valores financeiros de caráter suplementar efetuados em 10 parcelas mensais (de fevereiro a novembro) para a cobertura de 200 dias letivos, conforme o número de matriculados em cada rede de ensino e o tipo de escolaridade do aluno. Outra caracteristica é que parte do recurso do PNAE deve ser gasto na aquisição de de produtos da agricultura familiar, medida que estimula o desenvolvimento econômico e sustentável das comunidades. 
As instituicões de ensino que recebem os recursos do PNAE, tem feito manobras nas compras e doações dos gêneros alimentícios para as crianças matriculadas e que estão em suas casas em estudo remoto, em conformidade com a lei Lei nº 13.987, de 7 de abril de 2020 (Brasil, 2020). Assim, medidas diferentes tem sido adotadas pelas instituições de ensino da rede federal e essas receberam orientações sobre a execução do PNAE durante a pandemia (MAPA/MEC, 2020).

Atualmente, o valor repassado pela União a estados e municípios por dia letivo para cada aluno é definido de acordo com a etapa e modalidade de ensino, mostrado na tabela a seguir:

Tabela 1: Modalidade De Ensino X Valor Repassado Por Aluno.

\begin{tabular}{|l|l|}
\hline Etapa/Modalidade De Ensino & Valor Por Aluno \\
\hline Creches & $\mathrm{R} \$ 1,07$ \\
\hline Pré-Escola & $\mathrm{R} \$ 0,53$ \\
\hline Escolas Indígenas E Quilombolas & $\mathrm{R} \$ 0,64$ \\
\hline Ensino Fundamental E Médio & $\mathrm{R} \$ 0,36$ \\
\hline Educação De Jovens E Adultos & $\mathrm{R} \$ 0,32$ \\
\hline Ensino Integral & $\mathrm{R} \$ 1,07$ \\
\hline Escolas De Ensino Médio Em Tempo Integral & $\mathrm{R} \$ 2,00$ \\
\hline Atendimento Educacional Especializado No Contra turno & $\mathrm{R} \$ 0,53$ \\
\hline
\end{tabular}

Fonte: Autores.

O isolamento social impõe importantes mudanças socioculturais, redução da atividade física e alterações nos hábitos alimentares, determinantes consideráveis do estado nutricional. As iniciativas de educação alimentar e nutricional (por meio de programas educacionais na televisão, virtual ou rádio) devem ser realizadas de forma, que orientem e estimulem a adoção/manutenção de hábitos alimentares saudáveis para toda a família (Ribeiro-Silva, et al, 2020).

É sabido que a pandemia alterou muitas dinâmicas sociais familiares e outras tantas mudanças precisam ser feitas. Lemos, Barbosa e Monzato (2020) publicaram um trabalho relatando as situações das mulheres em trabalho remoto. Observaram que a demanda de trabalho pelas mulheres foi aumentada, não necessáriamente o trabalho, mas houve a incorporação de funções domésticas. As mulheres mães solteriras relataram maior cansaço em relação às mães casadas, que estão tendo ajuda de seus maridos, essas apesar do cansaço relataram que estão satisfeitas na conciliação trabalho-família, mas o aspecto alimentação e nutrição não foi evidenciado.

Do exposto, o presente trabalho teve por objetivo o levantamento de publicações familiares, de responsáveis, sejam reclamações e relatos de dificuldades que tiveram sobre a elaboração e oferta de alimentos saudáveis a seus filhos em idade infantil, que vai da aquisição até o momento do preparo devido as novas rotinas tendo que ser adaptadas. Além, do levantamento, houve a busca de publicações sobre as soluções dos problemas publicados.

\section{Metodologia}

A organização do trabalho se deu pela verificação de mudanças abruptas da sociedade, em que mães e responsáveis, saíram de seus ambientes profissionais e voltaram para suas residências para o home officer. Uma vez em casa, além de trabalhar, reassumiram suas atividades domesticas como responsáveis e uma dessas funções é o preparo de refeições a seus filhos.

Portanto, foi objetivado o levantamento desses relatos publicados na internet, de mães que em trabalho remoto estão com atividades domestícas e sentindo dificuldades no preparo e na oferta de alimentos saudáveis a sesu filhos e familiares. 
A busca por publicações foi livre, de artigos, de reportagens em jornais ou em revistas ou mesmo publicações em blogs e posts, pela plataforma do Google e Google Acadêmico e a indexixação utilizada foi "mães trabalho remoto alimentação" e "fome oculta infantil no Brasil" intervalo de 2020 a 2021, por serem consequencias do quadro de Pandemia COVID-19.

\section{Resultados e Discussões}

No levantamento bibliográfico realizado pela plataforma Google Acadêmico no período de 2020 a 2021 e usando a indexação "mães trabalho remoto alimentação" foram listadas 1.560 artigos publicados, e com a inexação "fome oculta infantil no Brasil" foram listadas 1.340 publicações.

Um trabalho se destaca sobre a alimentação de crianças em tempo de pandemia, elaborado por Assunção e colaboradores (2020) onde realizaram uma cartilha denominada "Vamos brincar de cozinhar?" onde foram dadas dicas de receitas para serem realizadas com crianças, receitas que podem ser realizadas com crianças durante o período de isolamento social, com o objetivo de promover uma interação das crianças com os alimentos, possibilitando uma atividade sensorial e estimulando uma alimentação saudável. O trabalho foi informativo sobre a alimentação para os responsáveis terem algo, para iniciar uma orientação nutricional.

Ademais, os artigos publicados não apresentaram reclamações de mães ou responsáveis e as dificuldades alimentares pelas crianças, dessa forma foi realizada a busca ampliada de publicações que não são artigos científicos e foram identificados 3.260 .000 resultados.

Focando a alimentação, a reportagem de Correio Brasiliense (2020) denominada "Mães conciliam filhos, aulas online, casa e home office durante o isolamento" traz o relato de uma mãe em trabalho remoto que diz dividir as responsabilidades dela de casa com o parceiro, que ele dá banho, troca de fraldas e coloca para dormir, mas que ela não abre mão de disciplinar os horários das atividades em casa e do preparo de alimentações saudáveis.

A reportagem da Revista IHU online (2020) traz um contraste na reportagem de Edelberto Behs titulada "Mães jornalistas em teletrabalho sentem-se esgotadas", em uma pesquisa realizada pela revista de 7 a 17 de agosto, respondida por 629 profissionais de todos os Estados e do Distrito Federal e divulgada no Dia Internacional da Igualdade Feminina de 2020, constatou que $85,9 \%$ das colegas se sentem sobrecarregadas, ainda que compartilhem cuidados com aulas online, alimentação e cuidados da casa, ao mesmo tempo em que precisam conciliar o trabalho home office ou presencial.

Na reportagem publicada na Revista online Migalhas, denominada "O horário de almoço não é feito para cozinhar" de 2021 e escrito pela Bianca Heena dos Santos, relatou ter tido uma pesquisa encomendada pela empresa Bepantol Babys para o IBOPE, relaizada com mil pessoas, as quais $84 \%$ afirmaram que a alimentação familiar, o preparo das refeições, é de responsabilidade exclusiva da figura da mãe. O porém é que a atividade de preparo não é apenas preparo, envolve: fazer a lista de compras, ir ao supermercado, guardar as compras e higienizá-las, separar os insumos a serem usados no preparo, o preparo propriamente dito com a limpeza da cozinha após a refeição. Todas as ações significam tempo e disposição, por mais que algumas das ações descritas não ocorram.

No site Brasil de Fato, houve uma reportagem de Lu Sudré (2021) denominada "Sobrecarga, desemprego e insegurança: a vida das mães um ano após chegada da Covid-19", traz o relato de uma mãe criticando os altos preços dos alimentos e impostos que estão sendo aplicados e cobrados num período de fragilidade social e economica de enfrentamento da pandemia.

Se não bastasse o cansaço pela sobrecara de funções, as mães, responsável pelas refeições familiares com trabalho remoto, ainda precisam equilibrar os recursos financeiros, que se mostram escassos. Dessa forma, o site a Adusp (2021) publicou a reportagem "Mães da escola Aplicação exigem merenda escolar", onde as mães precisando de ajuda recorrem às escolas fechadas pelo direito à merenda escolar para seus filhos matriculados na instituição.

Atitudes como a descrita, iniciaram um processo das instituições publicas, que recebem recursos federais do PNAE, sobre a aquisição de cestas básicas que foram distribuidas às famílias de crianças matriculas e que estão no ensino remoto, como 
por exemplo, a ESEBA da UFU, Escola de Educação Básica da Universidade Federal de Uberlândia, que nos dias de 20 a 25 de abril de 2021 realizou a distribuição de cestas básicas.

A Insegurança Alimentar (IA) ficou mais crítico em 2020, em função da pandemia da Covid-19, especialmente para a população pediátrica, como evidenciam os dados da UNICEF.11 A pesquisa realizada no segundo semestre de 2020 aponta que $61 \%$ das famílias com crianças e adolescentes apresentaram redução de renda, sendo mais evidente nas famílias mais pobres (69\%). O aumento do consumo de alimentos industrializados, refrigerantes e alimentos preparados em fast food durante a pandemia foi maior nas famílias com crianças e adolescentes e relatado por 54\% dos entrevistados, segundo estudos do Grupo De Trabalho Dos Mil Dias Da Sociedade Pediatrica De SP - por: Paula, Micheloni (2021).

Segundo um levantamento da Revista Eletrônica Acervo Saúde (2020), as implicações desta súbita quebra de paradigmas são amplas devido ao isolamento social recorrente da Pandemia COVID - 19 repercutem nos diversos âmbitos de interação social. Entre elas, a epidemia de obesidade precisa ser considerada e as implicações nesse cenário devem ser avaliadas. Um recorte possível seria a obesidade entre crianças e adolescentes, de várias formas favorecida durante o distanciamento social imposto como estratégia de enfretamento à disseminação da COVID - 19. Além dos prejuízos físicos, é necessário considerar os possíveis prejuízos psicológicos decorrentes do excesso de peso, como desenvolvimento de depressão, ansiedade, distúrbios do sono, baixa autoestima, imagem corporal distorcida, rendimento escolar aquém do esperado e dificuldades de relacionamento.

Apesar dos esforços para a destinação correta dos alimentos adquiridos com os recursos do PNAE às familias que precisam, polticamente houveram desgastes sobre o recurso no enfraquecimento de à agricultura familiar e nos gêneros alimentícios adquiridos com o recurso e repassado, como os alimentos ultraprocessados que não são reconhecidos como alimentos saudáveis, esses aspectos são descritos na reportagem "Como o desmonte do PNAE compromete a segurança alimentar infantil", publicado no site Lunetas em 2021 e escrtio o texto por Ariela Doctors.

\section{Conclusão}

Segundo a Sociedade Brasileira de Pediatria (2020), as estratégias para conter a disseminação da COVID-19 impactam diretamente na alimentação, já que a restrição da mobilidade limita a frequência de compras de gêneros alimentícios. A consequência é um maior consumo de alimentos processados e enlatados que são mais fáceis de adquirir e armazenar e possuem maior prazo de validade. Esse tipo de alimento tem baixo valor nutricional e alto valor calórico, contribuindo para o surgimento ou agravamento de sobrepeso e obesidade em crianças e adolescentes e outro fator que impacta diretamente na epidemia de obesidade é a redução das atividades físicas, fortemente influenciada pela interrupção das aulas presenciais, já que a escola é ambiente propício para as interações grupais ativas, realização de esportes e atividades recreativas.

Com relação as diversas atividades que implicam aglomeração de pessoas, como as aulas presenciais, estão paralisadas, exigindo de todos os envolvidos a criação de estratégias para reinventar a educação e permitir que ela alcance seu papel da forma mais inclusiva possível (Ornell, et al., 2020).

A interrupção das aulas presenciais, além de todos os possíveis impactos na formação dos estudantes, gera também uma lacuna no que se refere à alimentação, já que a merenda escolar é fonte segura e equilibrada de alimentação, sendo, em alguns casos, a única refeição com essas características acessível a vários alunos. Na situação econômica atual, o sustento de muitas famílias ficou comprometido e a disponibilidade de alimentos nutritivos ficou ainda mais escassa para as crianças e adolescentes em situação de vulnerabilidade socioeconômica. Nessas famílias, a contribuição da escola na alimentação fica ainda mais evidenciada e a impossibilidade de desfrutá-la pode gerar prejuízos no crescimento e desenvolvimento de inúmeras crianças e adolescentes (Ornell, et al., 2020). 
Além disso, muitas crianças não dispõem de local adequado para brincadeiras ou realizações de atividades que permitam a cessação do sedentarismo (Cabrera, Aguilar, Jaramillo et al.,2020).

As aulas de apoio e as academias estão com atividades suspensas o que também contribui para a manutenção de altos níveis de inatividade. A obesidade está comprovadamente relacionada à desregulação da resposta imune, tornando o indivíduo susceptível a infecções. Em pacientes com COVID - 19, a obesidade pode causar problemas respiratórios e dificuldade de intubação traqueal, aumentando risco de complicações e mortalidade (Sociedade Brasileira De Pediatria, 2020).

Deste modo, para evitar o desenvolvimento ou agravamento da obesidade e evitar as complicações decorrentes dela, a família deve proporcionar um ambiente saudável e seguro, fornecendo apoio emocional, priorizando alimentos nutritivos, estimulando as atividades físicas e limitando o tempo de tela. Essas medidas são necessárias tanto para evitar o excesso de peso e suas repercussões à longo prazo, quanto para suplantar o aumento do risco associado à obesidade caso essas crianças e adolescentes sejam expostos à infecção pelo COVID - 19 (Sociedade Brasileira De Pediatria, 2020).

\section{Considerações Finais}

Levando em consideração o levantamento realizado anteriormente nota-se um dos maiores desafios para uma alimentação saudável tem grande peso com relação a nova rotina vivida devido a pandemia COVID-19, pois demanda tempo dos responsáves para o preparo dos alimentos, além de uma mudança de hábitos alimentares mais saudável, identificamos uma participação maior das crianças neste processo de reeducação alimentar envolvendo elas nas atividades de preparo do seu alimento.

Este processo demanda tempo dos responsáveis, sendo ainda um ponto critico, pois devem se organizar entre o trabalho (home office/presencial), afazeres de casa, educação infantil, administração dos recursos financeiros para adquirir os alimentos necessários e suprir toda demanda financeira atual. Mesmo havendo um empenho por parte das políticas públicas criadas e adaptadas durante esse período de pandemia COVID-19 para auxiliar na alimentação das crianças de baixa renda, solucionado somente a questão financeira com relação a alimetação infantil, porém, a sobre carga, relatada principalmente por mães solteiras ainda é um problema, que afeta a rotina diária sem visão de melhora, devido o aumento da demanda home office e a necessidade de manter a renda familiar para suprir as outras demandas financeiras da casa, além de todas as atividades exercidas.

Mas apesar de toda dificuldade apresentada ao longo dos anos de 2019 e 2020 o quadro da pandemia COVID - 19 no Brasil fica cada vez mais agravante afetando diretamente o formato de vida brasileiro, sendo necessário adaptar-se de acordo com suas necessidades, e o governo vem auxiliando com os projetos para garantir uma alimentação básica e saudável, com auxilio financeiro e também através de kits alimentação distribuidos nas comunidades, podendo assim ser utilizado para auxiliar na alimentação infantil das comunidades carentes.

\section{Referências}

Adusp; Mães Da Escola De Aplicação Exigem Merenda Escolar, https://www.adusp.org.br/index.php/105-defesa-da-universidade/condicoes-de-trabalho/1142maes-da-escola-de-aplicacao-exigem-merenda-escolar

Brasil: Ministério Da Saúde (Ms). Recomendações De Alimentação Em Tempos De Pandemia De Covid -19 - Brasília - df, 2020.37 p.

Brasil: Ministério Da Saúde (MS). Fundo Nacional De Desenvolvimento Da Educação (FNDE) - from https://www.fnde.gov.br/index.php/programas/pnae/pnaesobre-o-programa/pnae-sobre-o-pnae

Brasil. Diário Oficial Da União. Publicado em: 07/04/2020| Edição: 67-B | Seção: 1 - Extra | Página: 9. Órgão: Atos do Poder Legislativo: Lei no 13.987, de 7 de abril de 2020. Altera a lei n ${ }^{\circ} 11.947$, de 16 de junho de 2009, (PNAE). $1^{\text {a }}$ edição. Ano 2020 tiragem: digital elaboração, distribuição, informações: Ministério Da Agricultura, Pecuária E Abastecimento Secretaria De Agricultura Familiar E Cooperativismo Departamento De Cooperativismo, from https://www.in.gov.br/en/web/dou/-/lei-n-13.987-de-7-de-abril-de-2020-251562793

Behs E. (2020), Mães Jornalistas Em Teletrabalho Sentem-Se Esgotadas - Revista IHU On-Line 28 agosto 2020, from http://www.ihu.unisinos.br/78noticias/602311-maes-jornalistas-em-teletrabalho-sentem-se-esgotadas 
Cabrera M. P. C., Aguilar J. L. L., \& Jaramillo L. M. T., (2020). A Pandemia De Covid-19 E Suas Repercussões Na Epidemia Da Obesidade De Crianças E Adolescentes. Revista Eletrônica Acervo Saúde. https://acervomais.com.br/index.php/saude/article/download/4743/3392/.

Costa Lemos A.H.; Barbosa A.O.; \& Monzato P. P; RAE - Revista De Administração De Empresas. Covid-19 E As Configurações Do Conflito Trabalho-Família: Mujeres En Home Office Durante La Pandemia De Covid-19 Y Las Configuraciones Del Conflicto Trabajo Y Família ; RAE - revista de administração de empresas. Fórum • rev. Adm. Empr rae | são paulo | v. 60 | n. 6 | nov-dez 2020 ,from https://doi.org/10.1590/s0034-759020200603

Doctors A. (2021). Como O Desmonte Do Pnae Compromete A Segurança Alimentar Infantil - Lunetas 19.05.2021, from https://lunetas.com.br/desmontepnae-compromete-seguranca-alimentar-infantil/

Fellet J. (2020) Bbc News - Piora Da Alimentação Na Pandemia Deixa População Mais Vulnerável À Covid-19 - @ joaofellet; BBC News Brasil Em São Paulo 16 maio 2020, from https://www.bbc.com/portuguese/internacional-52626216

Helena B. F., O Horário De Almoço Não É Feito Para Cozinhar - Migalhas 11 de março de 2021, from https://www.migalhas.com.br/depeso/341615/o-horariode-almoco-nao-e-feito-para-cozinhar

Ornell F. (2020), Pandemia De Medo E Covid-19: Impacto Na Saúde Mental E Possíveis Estratégicas. Revista Debates In Psychiatry. https://d1wqtxts1xzle7.cloudfront.net/63220777/PandemiademedoeCOVID-19impactona20200506-102677-146aa84-with-cover-page.pdf?Expires= 1622 818 819\&Signature=O2TYoEy7ZWS7KvmEEUv6Waj87Hbhc $\sim 88 B 5 \mathrm{mSshndcIb2} \mathrm{3 \sim WYXNNd97Gpw4vsbGJhuSL7yBPCBw2AY97FqDqukhoW9vQS071W4}$ CUeo2QZi6MTjSv DPor6B5-g weLTNjWnDcZtXwBM79IXKhUy-hhg6x3xIIHxM6ZoGHC8cHxGbGT0 LkFEm9d5nCy0188NY TpHKOTDwX9Jat AVz JXKy4XjDBbv XfKYiYFMvpq DDr19KC50s9nZJ-y6J9mJfPf6F81jO74sOjjXqTz7bVP75SdKN4Y-gxMmVwowUenzv3vCMjzfg3NPwOBPAzY PrFsu biR T ZiTgbSuHyiKPZXbaA_\&Key-Pair-Id=APKAJLOHF5GGSLRBV4ZA

Paula M. A.; \& Micheloni P. E. I. (2021) Insegurança Alimentar Na Gestante E $1^{\text {a }}$ Infância: Impactos Nos Primeiros Mil Dias. Grupo De Trabalho Dos Mil Dias Da SPSP, from https://www.spsp.org.br/PDF/GT\%20Mil\%20Dias_Inseguran\%C3\%A7a\%20Alimentar_Final.pdf

Prefeitura De Uberlândia; Programas - Pro-Pão, from https://www.uberlandia.mg.gov.br/prefeitura/secretarias/agronegocio-economia-e-inovacao/programasagropecuaria-uberlandia/

Prefeitura De Uberlândia; Programas - Kit Alimentação, from https://www.uberlandia.mg.gov.br/prefeitura/secretarias/educacao/kit-alimentacao-escolar/

Revista Senado (2020): Proposta Garante Auxílio Financeiro Para Alimentação De Crianças Da Rede Pública De Ensino - da redação |07/04/2020, https://www12.senado.leg.br/noticias/materias/2020/04/07/proposta-garante-auxilio-financeiro-para-alimentacao-de-criancas-da-rede-publica-de-ensino

Ribeiro T (2020). Jornal Folha De São Paulo - Refeição Das Crianças Pode Ser Um Fator De Ansiedade Para Pais.https://www1.folha.uol.com.br/comida/2020/04/refeicao-das-criancas-pode-ser-um-fator-de-ansiedade-para-pais.shtml

Ribeiro-Silva R. C., Pereira M., Campello T., Aragão E., de Medeiros Guimarães J.M., Ferreira A. J. F., Barreto M. L., Chaves dos Santos S. M., Revista Ciência Saúde Coletiva: Implicações Da Pandemia Covid-19 Para A Segurança Alimentar E Nutricional No Brasil. Revista Ciênc. Saúde Coletiva, 25 (9), 3421 - 3430, from https://www.scielo.br/j/csc/a/mFBrPHcbPdQCPdsJYN4ncLy/?lang=pt

SENAC (2020): Portal Especializado Em Desenvolvimento Sustentável - Impactos Financeiros Na Pandemia: Fome, Trabalho Infantil E Mendicância - por setor 320 de julho de 2020, from http://setor3.com.br/impactos-financeiros-na-pandemia-fome-trabalho-infantil-e-mendicancia/

Silva T,, (2020) Mães Conciliam Filhos, Aulas Online, Casa E Home Office Durante Isolamento - Correio Brasiliense. https://www.correiobraziliense.com.br/app/noticia/revista/2020/05/10/interna_revista_correio,850826/maes-conciliam-filhos-aulas-online-casa-e-home-officedurante-isolam.shtml

Sobreira C. F. L.; César G.S.; Constâncio M. M.; Augusto V. A. S.; Regina P. G., A Pandemia De COVID - 19 E Suas Repercussões Na Epidemia Da Obesidade De Crianças E Adolescentes, from https://acervomais.com.br/index.php/saude/article/view/4743/3392

Sociedade Brasileira De Pediatria. Obesidade Em Crianças E Adolescentes E COVID - 19. Nota de Alerta. Sociedade Brasileira de Pediatria. Grupo de Trabalho em Atividade Física. https://www.sbp.com.br/imprensa/detalhe/nid/obesidade-em-criancas-e-adolescentes-e-covid-19/

Sudré L., Sobrecarga, Desemprego E Insegurança: A Vida Das Mães Um Ano Após Chegada Da COVID-19; Brasil https://www.brasildefato.com.br/2021/05/09/sobrecarga-desemprego-e-inseguranca-a-vida-das-maes-um-ano-apos-chegada-da-covid-19 